\title{
Principal Clinical Factors Predicting Therapeutic Outcomes After Surgical Drainage of Postoperative Cheek Cysts: Experience From a Single Center
}

\author{
Sung-Woo Cho', $\cdot$ Hyun Jung Lim $\cdot$ Yoonjae Song ${ }^{1}$ Young Kang $\cdot$ Jae Hyun Lim ${ }^{1} \cdot$ Yung Jin Jeon ${ }^{1}$ Doo Hee Han ${ }^{1}$ \\ Tae-Bin Won ${ }^{1}$. Dong-Young Kim ${ }^{1} \cdot$ Hyun Jik Kim ${ }^{1}$ \\ ${ }^{l}$ Department of Otorhinolaryngology-Head and Neck Surgery, Seoul National University Hospital, Seoul National University College of Medicine, \\ Seoul; ' ${ }^{2}$ Department of Otorhinolaryngology-Head and Neck Surgery, Seoul National University Bundang Hospital, Seongnam, Korea
}

Objectives. Postoperative cheek cyst (POCC) is a late postoperative complication of radical maxillary sinus surgery including the Caldwell-Luc (C-L) operation. The present study aimed to evaluate the therapeutic outcomes of surgical treatment for POCC and to assess the clinical factors correlated to these outcomes.

Methods. This study included 57 patients (67 nostrils) diagnosed with POCC who underwent surgical drainage. The medical records of the patients were retrospectively reviewed for radiological findings, treatment modalities, residual symptoms, and recurrences.

Results. In total, 30 patients were male and 27 patients were female with a mean age of 55 years, and the patients were usually diagnosed with POCC 28.2 years after radical surgery. Endonasal endoscopic marsupialization was performed via inferior meatal antrostomy, and if possible, middle meatal antrostomy was performed at the same time. In patients with cysts that were difficult to reach using an endonasal endoscopic approach, additional open C-L approaches were performed. The median follow-up period was 19.4 months. Overall, adequate drainage and symptomatic relief were achieved in 91\% (61/67) of the patients. The recurrence rate was significantly higher in patients who had anterolateral POCC. Failure to achieve symptomatic relief was correlated to a smaller cyst and the use of the open C-L approach for drainage.

Conclusion. The location and size of the cyst as well as the use of the open surgical approach were important factors in predicting the therapeutic outcome of POCC. The time point of treatment and surgical approaches should be based on the above-mentioned findings.

Keywords. Maxillary Sinus; Mucocele; Drainage; Treatment Outcome

\section{INTRODUCTION}

Postoperative maxillary cyst, sometimes known as postoperative cheek cyst (POCC) in Korea, is a late complication of radical

\footnotetext{
- Received April 9, 2018

Revised July 24, 2018

Accepted August 1, 2018

- Corresponding author: Hyun Jik Kim

Department of Otorhinolaryngology-Head and Neck Surgery, Seoul

National University College of Medicine, 103 Daehak-ro, Jongno-gu,

Seoul 03080, Korea

Tel: +82-2-2072-2203, Fax: +82-2-766-2447

E-mail: hyunjerry@snu.ac.kr
}

maxillary sinus procedures that are usually performed via Caldwell-Luc (C-L) surgery [1]. Approximately $20 \%$ of POCCs occur after radical maxillary surgery, and they develop 10-30 years after surgery $[1,2]$. Although POCC is rarely reported in Western countries, it is quite common in Asian countries, including Japan [3], and is the most common cause of maxillary mucocele in Korea [1,4].

In the C-L operation, the mucosa lining of the maxillary sinus is eradicated in the context of treating maxillary sinusitis [5]. However, although not fully understood, the remaining microscopic mucosal tissues or mucosal growth from the nasal cavity into the maxillary sinus may be responsible for the development

Copyright @ 2019 by Korean Society of Otorhinolaryngology-Head and Neck Surgery

This is an open-access article distributed under the terms of the Creative Commons Attribution Non-Commercial License (http://creativecommons.org/licenses/by-nc/4.0)

which permits unrestricted non-commercial use, distribution, and reproduction in any medium, provided the original work is properly cited. 
of POCC [6]. The most common symptoms are cheek pain, swelling, and nasal obstruction. However, symptoms can vary depending on the extent of the disease (from the absence of symptoms to the presence of orbital or dental symptoms) $[7,8]$.

Two approaches are used for treating POCCs. One is an open approach with a revised C-L operation, which requires the complete excision of the mucocele lining through the inferior nasoantral window [9]. The other is an intranasal approach involving complete marsupialization using the recent advanced trans-nasal endoscopic approach along with middle meatal antrostomy (MMA) or inferior meatal antrostomy (IMA). However, if the location of the POCC is far anterior or lateral or if the bony wall of the inferior meatus is too thick, the marsupialization of the lesion can be performed via conventional surgery using the canine fossa approach (also known as the C-L approach) along with IMA at the end of the procedure to secure an internal (endonasal) drainage [6,7,10-13].

Recently developed instruments, including the surgical drill and navigation system, have expanded the indications for endoscopic endonasal surgery that make it possible to manage certain sinonasal mucoceles that are anatomically difficult to access using the endoscopic endonasal approach alone. However, even after performing surgical drainage via endoscopic marsupialization, POCC may recur in $2 \%-38 \%$ of patients [13]. In addition, patients may still complain about residual symptoms, such as cheek pain and repeated cheek swelling even after surgical treatment and medication. This may be correlated to the development of early postoperative complications, such as buccal hematoma. However, recently, we have experienced patients with a patent drainage still complaining of cheek pain 3 months after surgery. Therefore, further investigation must be conducted to understand the predictive factors associated with the therapeutic outcomes of POCC and indications for surgery.

In this study, we evaluated the therapeutic outcomes of endonasal marsupialization by analyzing disease recurrence depending on the anatomical location of POCCs. Moreover, the change in clinical symptoms after endoscopic marsupialization was investigated to identify the therapeutic outcomes of a successful POCC surgical drainage.

\section{H}

- Postoperative cheek cyst is a late complication of radical maxillary sinus procedures, including the Caldwell-Luc surgery.

- The location (anterior and lateral) of the cyst was correlated to recurrence after drainage.

- The size (small) of the cyst and an open surgical approach are important factors correlated to the residual symptoms observed even after adequate drainage.

\section{MATERIALS AND METHODS}

\section{Participants}

Patients with POCC who had been surgically treated from January 2011 to January 2016 at a single center were recruited, and their medical records were retrospectively reviewed. POCC was confirmed based on the patient's history of previous C-L surgery, symptoms of facial swelling or pain, presence of a lesion on computed tomography (CT) scan, and pathological examination results after surgical treatment. Those without imaging or other proven pathological examination results were excluded. Patients who had been previously treated surgically before the study period or those who had been treated at other institutions during the study period were also excluded.

Age at the time of operation, surgical approach, follow-up period, and treatment outcomes were analyzed using clinical data. Treatment outcomes were evaluated based on the decrease in the degree of symptoms and patency of the POCC ostium on endoscopy. According to preoperative CT images, the size, number, and location of the cyst were evaluated, and the maximal diameter was measured from the axial image. Postoperative CT scans were performed to assess the recurrence of POCC in selected cases, such as in patients with obliterated newly formed ostium or those with a patent ostium but still presented with symptoms. This study was approved by the Institutional Review Board of Seoul National University Hospital (IRB No. SNUH 1806-022-949). Informed consents from patients were not required as no images are identifiable.

\section{POCC classification}

For the localization of the cyst, we modified the classification system originally developed by Nakamura et al. [14]. In the

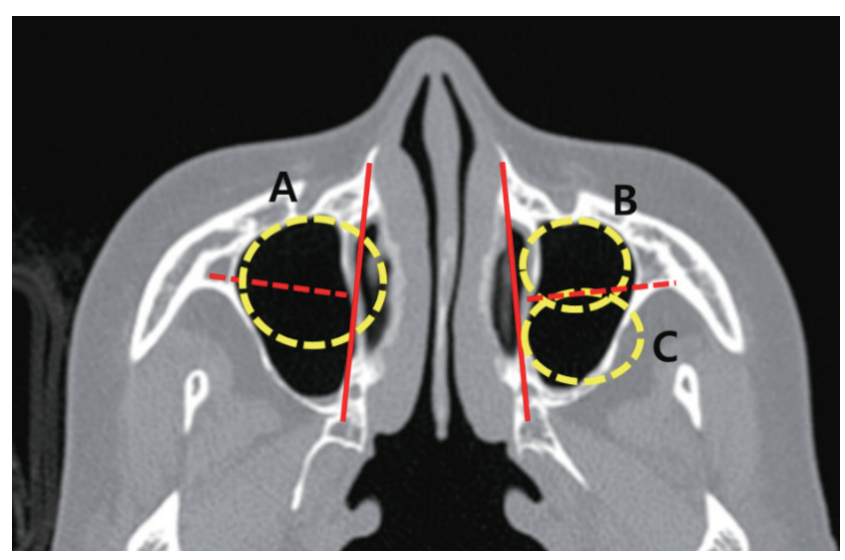

Fig. 1. Classification of postoperative cheek cysts. In the conventional system, a line is drawn between the anterior end of the inferior turbinate and base of the lateral pterygoid process (red lines). A medial cyst $(A)$ extends toward the midline across this imaginary line, and a lateral cyst is classified as anterolateral (B) or posterolateral (C) based on the position of the center of the cyst with reference to another line (red dotted lines) that lies vertically to the initial line. 


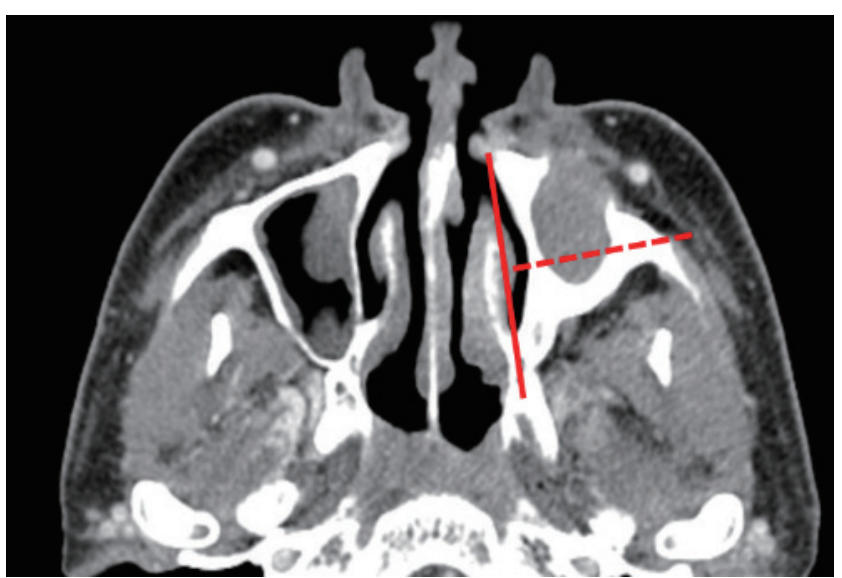

Fig. 2. Modified classification of postoperative cheek cysts. Line is drawn between the anterior end of the inferior turbinate and base of the lateral pterygoid process (red line). We modified the system such that an anterior cyst was defined as the posterior end of the cyst wall is located anterior to the midline (red dotted line). In cases where the posterior wall of the cyst is posterior to the midline, the cyst is classified as non-anterolateral.

original system, a line between the anterior end of the inferior turbinate and the base of the lateral pterygoid process on the axial CT plane at the mid-level of the inferior meatus can be drawn. A medial cyst extends toward the midline across this imaginary line, and a lateral cyst is subdivided anterolaterally or posterolaterally based on the position of the center of the cyst (Fig. 1). As many cases that were defined as anterolateral according to the original system could be approached via the endoscopic endonasal approach only, we modified the system such that an anterior cyst was defined as a cyst with a posterior end located anterior to the midline (Fig. 2). The cyst was considered as multiple cysts if there is a bony or membranous septation separating the cysts (Fig. 3).

\section{Surgical approach and outcome analysis}

All patients underwent endonasal marsupialization of the POCC cavity using a 4-mm rigid endoscope. When the lesion could not be accessed, the C-L approach along with endoscopic endonasal marsupialization was used. In both approaches, marsupialization of the cystic lesion was performed via IMA and/or IMA along with MMA if possible to induce physiological ventilation through the natural ostium of the maxillary sinus. A navigation system (Fusion; Medtronic, Minneapolis, MN, USA) had been used in selected cases presenting with either multiple cysts or cysts that are located anterolaterally. An endonasal drill with a 3.2-mm diamond burr had been used in cases with thick bony cystic wall and those that cannot be removed using routinely used endoscopic sinus surgery instruments.

The primary outcome was recurrence. POCC recurrence was defined as an occlusion in the ostium of the marsupialization on endoscopic examination or the presence of a cystic lesion on CT

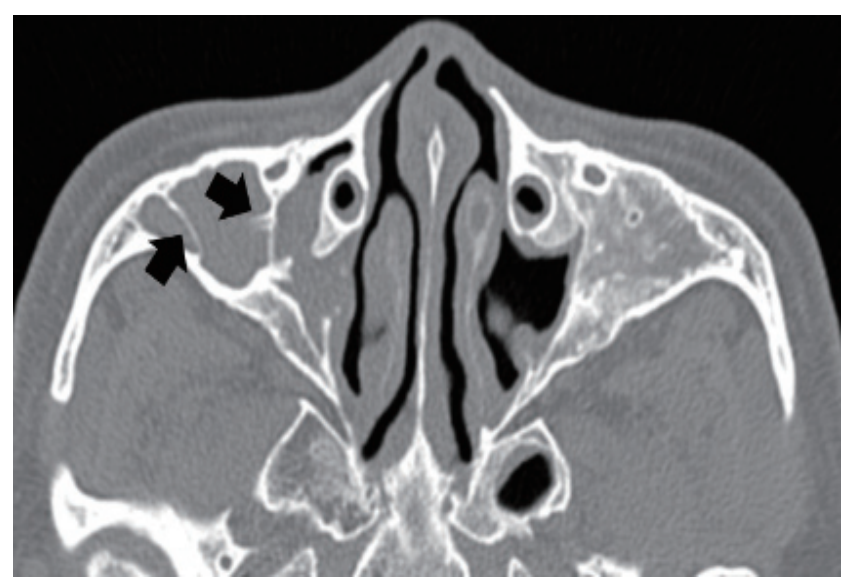

Fig. 3. Case of multiple cysts. Cysts separated by bony or membranous septations (black arrows) were considered as multiple cysts.

scan performed during the follow-up period. The secondary outcome was the presence of residual symptoms. Symptoms were evaluated at least 3 months after surgery in the presence of adequate openings into the nasal cavity.

\section{Statistical analysis}

Results are presented as mean \pm standard deviation or median with interquartile range (IQR). Statistical analysis was performed using the IBM SPSS ver. 22.0 (IBM Corp., Armonk, NY, USA). Cross-table analysis with Fisher exact test was used for categorical variables, whereas Mann-Whitney $U$-test was used for continuous nonparametric variables. Logistic regression analysis (forward: conditional method) was performed for the risk evaluation of the combination of factors. Multivariate Cox proportional hazard models, with the duration of recurrence as the underlying time metric, were used to estimate the risk of POCC recurrence associated with the factors. The relapse-free survival curves of cyst recurrence were obtained using the Kaplan-Meier method. The $P$-value $<0.05$ was considered statistically significant.

\section{RESULTS}

\section{Patient demographics and subjective symptoms}

In total, 57 patients (male:female, 30:27; 67 nostrils) with POCC who underwent surgery were included in this study. The mean age of the patients was $55 \pm 11$ years, and the mean duration of the development of POCC was $28.2 \pm 9.1$ years. The median duration of the follow-up after surgery was 19.4 months (IQR, 24.0 months; range, 1.1 to 87.9 months) (Table 1 ). In total, $85.1 \%$ of the patients complained of cheek pain, and $50.7 \%$ of the patients presented with cheek swelling. Moreover, $9.0 \%, 6.0 \%$, $4.5 \%$, and $1.5 \%$ of the patients had dental pain or dental discharge, orbital pain, nasal obstruction, and headache, respec- 
Table 1. Patient demographics and initial characterization of the cyst and surgical approaches

\begin{tabular}{lc}
\hline Parameter & Value \\
\hline Age at the time of diagnosis (yr) & $55 \pm 11$ \\
Male:female (male:female per nostril) & $30: 27$ (34:33) \\
Mean size (mm) & $34.7 \pm 7.9$ \\
Duration of development (yr) & $28.2 \pm 9.1$ \\
Location of the cyst (anterolateral:non-anterolateral) & $12: 55$ \\
Septation of the cyst (single:multiple) & $32: 35$ \\
Surgical approach (endoscopic endonasal only: & $62: 5$ \\
$\quad$ combined with the C-L approach) & \\
Duration of follow-up (mo) & $19.4(24.0)$ \\
\hline
\end{tabular}

Values are presented as mean \pm standard deviation or median (interquartile range).

C-L, Caldwell-Luc.

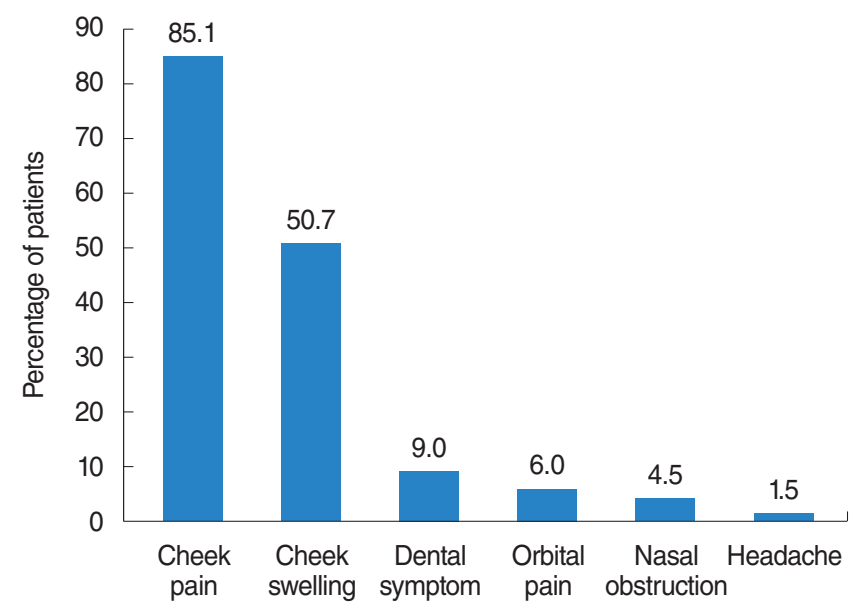

Fig. 4. Initial presenting symptoms of postoperative cheek cysts. Cheek pain and swelling were the primary symptoms, followed by dental symptoms, orbital pain, nasal obstruction, and headache. The categories herein are not mutually exclusive.

tively (Fig. 4).

\section{Characteristics of the cyst and surgical options}

The mean diameter of the cyst was $34.7 \pm 7.9 \mathrm{~mm}$. POCCs that are located anterolaterally were observed in 12 cases (17.9\%), and the remaining 55 cysts $(82.1 \%)$ were in a non-anterolateral position (medial or posterolateral). Thirty-two cases of POCC were unilocular $(47.8 \%)$, and the other 35 cases $(52.2 \%)$ were multilobular. An endoscopic endonasal approach was used in 62 cases $(92.5 \%)$, and the C-L approach along with an endoscopic endonasal approach was performed in five cases $(7.5 \%)$. Fourteen patients $(20.9 \%$ ) had concurrent chronic rhinosinusitis (two cases with nasal polyps); thus, they underwent functional endoscopic sinus surgery at the same time. In 39 cases (58.2\%), IMAs without MMA were performed for endoscopic marsupialization primarily due to a contracted middle meatus, and in the other 28 cases $(41.8 \%)$, endoscopic marsupialization was performed via IMA along with MMA.
Table 2. Univariate comparison of each parameter in accordance to cyst recurrence

\begin{tabular}{lccr}
\hline Parameter & $\begin{array}{c}\text { No recurrence } \\
(n=61)\end{array}$ & $\begin{array}{c}\text { Recurrence } \\
(\mathrm{n}=6)\end{array}$ & P-value \\
\hline Age (yr) & $55 \pm 11$ & $62 \pm 10$ & 0.135 \\
Male:female & $32: 29$ & $2: 4$ & 0.322 \\
Size (mm) & $34.7 \pm 7.8$ & $35.5 \pm 9.6$ & 0.740 \\
Duration of development (yr) & $28.4 \pm 9.4$ & $26.3 \pm 5.5$ & 0.789 \\
Location (anterior and lateral) & 7 & 5 & $<0.001$ \\
Multiple cysts & 29 & 6 & 0.016 \\
Surgical approach (combined with & 4 & 1 & 0.384 \\
$\quad$ the C-L approach) & & & \\
Concurrent chronic sinusitis & 13 & 1 & 0.633 \\
\hline
\end{tabular}

Values are presented as mean \pm standard deviation.

C-L, Caldwell-Luc.

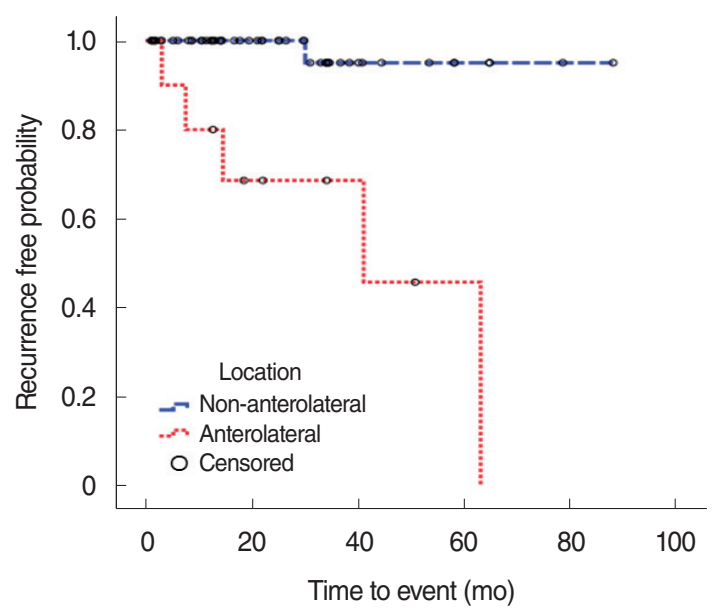

Fig. 5. Kaplan-Meier probability curves for the risk of cyst recurrence. According to the location of the cyst, the recurrence rates were significantly different (log-rank test, $P$-value $<0.001$ ).

\section{Therapeutic outcomes of POCC and its recurrence}

Recurrence of cystic lesions occurred in six patients $(9.0 \%)$. Of these patients, three had persistent symptoms, such as facial swelling or cheek pain, after the marsupialization of POCC. Two patients underwent endoscopic marsupialization along with the C-L approach, and two other patients were lost to follow up. The remaining two patients did not complain of any symptoms associated with recurrent cystic lesions, and further surgical treatment was not recommended. The mean duration until the recurrence of cystic lesion treated with endoscopic marsupialization was $26.5 \pm 22.9$ months. Among the recurrent cases, the cyst was located anterolaterally (5/6 vs. $7 / 61, P<0.001$ ), and multiple cysts ( $6 / 6$ vs. $29 / 61, P=0.016)$ were more frequently observed in recurrent cases than in those without recurrence. Sex, age, size of the cyst, surgical approach, and presence of chronic sinusitis were not significantly different between recurrent cases and those without recurrence (Table 2). However, multivariate Cox proportional hazards analysis for cyst recur- 
Table 3. Univariate comparison of each parameter according to the remaining symptoms after adequate drainage

\begin{tabular}{lccc}
\hline Parameter & $\begin{array}{c}\text { Resolved } \\
\text { symptom } \\
(\mathrm{n}=52)\end{array}$ & $\begin{array}{c}\text { Remaining } \\
\text { symptom } \\
(\mathrm{n}=5)\end{array}$ & $P$-value \\
\hline Age $(\mathrm{yr})$ & $57 \pm 11$ & $61 \pm 12$ & 0.593 \\
Male:female & $29: 23$ & $1: 4$ & 0.145 \\
Size (mm) & $35.7 \pm 7.5$ & $27.6 \pm 3.3$ & 0.015 \\
Duration of development (yr) & $28.3 \pm 9.4$ & $35.4 \pm 9.8$ & 0.177 \\
Location (anterior and lateral) & 8 & 1 & 0.591 \\
Multiple cysts & 28 & 1 & 0.165 \\
Approach (C-L+IMA) & 3 & 2 & 0.056 \\
Concurrent chronic sinusitis & 11 & 0 & 0.327 \\
\hline
\end{tabular}

Values are presented as mean \pm standard deviation.

C-L, Caldwell-Luc; IMA, inferior meatal antrostomy.

Table 4. Multivariate analysis of the risk factors associated with symptom persistence after adequate drainage

\begin{tabular}{lclc}
\hline Parameter & Adjusted OR & \multicolumn{1}{c}{$95 \% \mathrm{Cl}$} & $P$-value \\
\hline Size & 0.769 & $0.598-0.988$ & 0.040 \\
$\begin{array}{c}\text { Surgical approach } \\
\text { (combined approach) }\end{array}$ & 32.832 & $1.739-619.811$ & 0.020 \\
\hline
\end{tabular}

OR, odds ratio; $\mathrm{Cl}$, confidence interval.

rence showed that only the anterolateral location of the cyst was significantly different (hazard ratio, 21.533 ; $95 \%$ confidence interval $[\mathrm{CI}], 2.051$ to $185.421 ; P=0.005)$. The KaplanMeier curves for the cyst recurrence were evident according to cyst location (anterolateral vs. non-anterolateral: log-rank test, $P$-value $<0.001$ ) (Fig. 5).

In six of 67 cases $(9.0 \%)$, symptoms, particularly pain in the cheek area, persisted even after performing adequate drainage of the cyst. However, only five of 57 cases $(8.8 \%)$ were evaluated because 10 patients had been lost to follow up less than 3 months after the surgical drainage. None of them had any signs of infection, such as hyperemia or focal heat over the cheek area. These patients had remaining symptoms, although they had a patent ostium without evidence of the disease on followup CT scan; thus, the symptoms were assumed to be caused by other conditions. For patients with unresolved symptoms without evidence of recurrence, univariate comparison showed that the size of the cyst tended to be significantly smaller compared to those with resolved symptoms after surgery $(27.6 \pm 3.3 \mathrm{~mm}$ vs. $35.7 \pm 7.5 \mathrm{~mm}$, respectively, $P=0.015$ ) (Table 3 ). However, multivariable analysis revealed that a larger cyst had a protective effect (adjusted OR, 0.769; 95\% CI, 0.598 to $0.988 ; P=0.040$ ), and the combined C-L approach (adjusted OR, 32.832; 95\% CI, 1.739 to $619.811 ; P=0.020$ ) increased the risk of the remaining symptoms after adequate drainage (Table 4). Other factors, including age, sex, location, and the number of cysts, were not significantly different.
Table 5. Enhanced endonasal approach for anterolateral POCC

\begin{tabular}{llcc}
\hline $\begin{array}{l}\text { Anatomical } \\
\text { location of } \\
\text { the cyst }\end{array}$ & \multicolumn{1}{c}{ Study } & $\begin{array}{c}\text { C-L } \\
\text { approach }\end{array}$ & $\begin{array}{c}\text { Endonasal } \\
\text { approach }\end{array}$ \\
\hline Overall & Kwon et al. (1997) [8] & $23.1(6 / 26)$ & $76.9(20 / 26)$ \\
& This study & $8.1(5 / 62)$ & $91.9(57 / 62)$ \\
Anterolateral & & & \\
& Kwon et al. (1997) [8] & $50(4 / 8)$ & $50(4 / 8)$ \\
& This study & $25(3 / 12)$ & $75(9 / 12)$ \\
\hline
\end{tabular}

Values are presented as percent (number/total).

POCC, postoperative cheek cyst; C-L, Caldwell-Luc.

\section{DISCUSSION}

In this study, we reviewed the therapeutic outcomes of surgical treatment for POCC, focusing on disease recurrence and the remaining symptoms after treatment to understand the appropriate indications for intranasal endoscopic drainage of POCC. The successful treatment outcomes of POCC should include adequate drainage and symptomatic relief. This study has shown that adequate drainage is correlated to the anatomical location of the cyst. That is, patients with cysts that are located anterolaterally have a higher recurrence rate. Moreover, even after adequate drainage of the cyst, the use of the C-L approach or the presence of a small cyst is more likely not to relieve the symptoms.

Previously, surgical approaches that have been used for POCC were primarily open approaches, such as the C-L surgery. Endoscopic marsupialization has become the treatment of choice for POCC due to the development of new instruments and imageguided systems [13]. However, an effective marsupialization of cysts located far from the lateral nasal wall is difficult when an endonasal endoscopic approach is used. In such cases, C-L surgery has also been recommended. In addition, this approach has been recommended for POCCs with excessively thick medial bony wall [13].

The present study revealed that $92.5 \%$ of POCCs could be managed with endonasal endoscopic marsupialization, including IMA or MMA, and nine of 12 cases $(75 \%)$ with anterolateral cysts could be managed with an endoscopic endonasal approach only. The ratio of endonasal endoscopic marsupialization for anterolateral cysts has been enhanced in recent studies compared to those published approximately 20 years ago when endoscopic sinus surgery had just gained popularity in Korea (Table 5) [8]. We undertook the external approach for the treatment of POCC in a more limited manner. Furthermore, among the 35 cases with multiple cysts, $91.4 \%$ (32/35) could be managed by endonasal endoscopic marsupialization alone. We identified that both image-guided navigation system and bony drilling of the lateral nasal wall would be necessary for the endonasal marsupialization of anterolateral POCCs. In our series, the navigation 
system was used in 11 cases, all of whom had multiple cysts or had cysts that were located anterolaterally. The exact location of the point for trial of marsupialization can be identified via CT scan, and the cyst can be safely drained after bony wall drilling with a navigation system during operation. Surgeons can ensure that the lesions are drained properly using a navigation system, even in cases of multiple cysts, which can be difficult to evaluate when visualized only with a rigid endoscope.

Recurrence was observed in six cases, of which five had a closed or narrow IMA window. The other patient had a narrow but opened ostium and presented with a newly formed cystic lesion in the previous surgical site. Thus, recurrences are caused by closed antrostomy before the entire cavity had been mucosalized and externalized or newly formed cystic lesions (aberrant mucosalization).

Previously, it has been reported that IMA should be $1 \times 2 \mathrm{~cm}$ in size to maintain patency [15]. However, such large antrostomy could not be achieved using an endonasal approach, particularly in anterolateral POCCs. In such cases, the C-L approach might be helpful. However, we suggest that the C-L approach should not be routinely performed for anterolateral POCCs. In this study, among the five patients with POCCs who were treated with endonasal marsupialization along with the C-L approach, one patient still presented with recurrence on CT scan, with a newly formed cystic lesion with septation into a cyst even though the IMA was patent. Thus, even when open approaches are used, septation may occur after wide antrostomy. As in our study, the combined C-L approach was considered a risk factor for residual symptoms after adequate drainage. This is probably due to the injury in the infraorbital or superior alveolar nerve causing facial pain or paresthesia, which is commonly associated with the C-L approach [16]. Usually, these nerve injuries recover as the nerves either regrow or the area is reinnervated by the nearby nerves [17]. However, repeated surgical trauma, as in our cases, may lead to prolonged injury.

In our study, patients with small cysts are more likely to experience incomplete symptom relief after adequate marsupialization. A relatively small cyst may not cause compressive symptoms, such as pain, and there may be other causes of pain in such cases. In our series, one patient was diagnosed with cervical plexus neuralgia with symptomatic improvement after blocking the cervical plexus with local anesthesia. Thus, surgeons and patients should be aware that these symptoms may persist even after an adequate marsupialization in such cases.

Despite the lack of statistical significance, a trend toward increased recurrence in the presence of multiple cysts indicates that the septated cysts must be removed. The complete removal of the septations may prevent aberrant healing, which may cause newly formed septations after the operation. Therefore, POCC drainage should be as wide as possible, and the complete removal of fibro-osseous septations must also be carried out.

Based on these observations, we suggest that the endonasal endoscopic approach should be the initial treatment of choice for all cysts regardless of the location, and if a wide antrostomy cannot be created or the removal of septations is not possible, only the combined C-L approach should be considered. In addition, a residual symptom may be observed after using the C-L approach. For a small cyst causing minimal symptoms without any signs of infection, the wait-and-see period is recommended, and during this time, other causes of the symptoms should be evaluated. In such cases, surgery may be indicated when all other causes of the symptoms have been excluded.

The present study has limitations. First, we investigated the recurrence of POCC or residual symptoms after marsupialization based on the location of the POCC. However, the diameter of the antrostomy site was not measured due to the limitations in approaching this area. In addition, the medication history of the patients and the presence of other sinonasal diseases, such as allergic rhinitis, which may have affected the antrostomy closure, was not evaluated [18,19].

The incidence of POCC would decrease since C-L surgery is rarely performed nowadays. However, the C-L approach is still considered as an option for surgically refractory maxillary sinusitis. Our findings also emphasized the basic principles of mucocele surgery of other paranasal sinuses, which is commonly performed.

In summary, the location, size and number of septated cysts as well as an open surgical approach might be important factors in predicting the therapeutic outcomes of endoscopic marsupialization. The C-L approach should be used only when a larger IMA or the complete removal of bony septation is needed in patients with anterolateral POCCs. For those with relatively small cysts, symptoms may not resolve even after adequate marsupialization of anterolateral POCCs.

\section{CONFLICT OF INTEREST}

No potential conflict of interest relevant to this article was reported.

\section{ACKNOWLEDGMENTS}

This work was supported by Research Resettlement Fund for the new faculty of Seoul National University, Seoul, Korea.

\section{REFERENCES}

1. Kaneshiro S, NakajimaT, Yoshikawa Y, Iwasaki H,Tokiwa N.The postoperative maxillary cyst: report of 71 cases. J Oral Surg. 1981 Mar; 39(3):191-8.

2. Yamamoto H,Takagi M. Clinicopathologic study of the postoperative maxillary cyst. Oral Surg Oral Med Oral Pathol. 1986 Nov;62(5): 
544-8.

3. Nishioka M, Pittella F, Hamagaki M, Okada N,Takagi M. Prevalence of postoperative maxillary cyst significantly higher in Japan. Oral Med Pathol. 2005;10(1):9-13.

4. Lee KC, Lee NH. Comparison of clinical characteristics between primary and secondary paranasal mucoceles. Yonsei Med J. 2010 Sep;51(5):735-9.

5. Macbeth R. Caldwell, Luc, and their operation. Laryngoscope. 1971 Oct;81(10):1652-7.

6. Kubo I. A buccal cyst occurring after a radical operation of the maxillary sinus. Z Otol Tokyo. 1927;33:896-7.

7. Jang TY, Lee SJ, Kim KT, ChoiYS. Management of postoperative mucocele of the maxillary sinus through endoscopic approach. Korean J Otorhinolaryngol Head Neck Surg. 2005 Mar;48(3):314-9.

8. Kwon SH, Yang YS, Min YG. Surgical treatment and clinical study of postoperative maxillary cyst. Korean J Otorhinolaryngol Head Neck Surg. 1997 Aug;40(8):1115-21.

9. Yoshikawa Y, Nakajima T, Kaneshiro S, Sakaguchi M. Effective treatment of the postoperative maxillary cyst by marsupialization. J Oral Maxillofac Surg. 1982 Aug;40(8):487-91.

10. Busaba NY, Salman SD. Maxillary sinus mucoceles: clinical presentation and long-term results of endoscopic surgical treatment. Laryngoscope. 1999 Sep;109(9):1446-9.

11. Huang CC, Chen CW, Lee TJ, Chang PH, Chen YW, Chen YL, et al. Transnasal endoscopic marsupialization of postoperative maxillary mucoceles: middle meatal antrostomy versus inferior meatal antrostomy. Eur Arch Otorhinolaryngol. 2011 Nov;268(11):1583-7.

12. Saito T, Ikeda T, Kono Y, Ohtsubo T, Noda I, Saito H. Implications of endoscopic endonasal surgery for the treatment of postoperative maxillary mucoceles. ORL J Otorhinolaryngol Relat Spec. 2000 JanFeb;62(1):43-8.

13. Sawatsubashi M, Murakami D, Oda M, Komune S. Transnasal endoscopic surgery of post-operative maxillary cysts. J Laryngol Otol. 2015 Mar;129 Suppl 2:S46-51.

14. Nakamura K, Kitani S, Sato H, Yumoto E, Kawakita S, Aibara R. Endoscopic endonasal surgery for postoperative maxillary cyst. Nihon Jibiinkoka Gakkai Kaiho. 1995 Jun;98(6):984-8.

15. Lund VJ. Fundamental considerations of the design and function of intranasal antrostomies. Rhinology. 1985 Sep;23(3):231-6.

16. Low WK. Complications of the Caldwell-Luc operation and how to avoid them. Aust N Z J Surg. 1995 Aug;65(8):582-4.

17. Robinson SR, Baird R, Le T, Wormald PJ.The incidence of complications after canine fossa puncture performed during endoscopic sinus surgery. Am J Rhinol. 2005 Mar-Apr;19(2):203-6.

18. Thompson LD. Paranasal sinus mucocele. Ear Nose Throat J. 2012 Jul; 91(7):276-8.

19. Abdel-Aziz M, El-Hoshy H, Azooz K, Naguib N, Hussein A. Maxillary sinus mucocele: predisposing factors, clinical presentations, and treatment. Oral Maxillofac Surg. 2017 Mar;21(1):55-8. 